\title{
MiRNAs of peripheral blood as the biomarker of schizophrenia
}

Kuanjun $\mathrm{He}^{1 * \dagger}$, Chuang Guo ${ }^{1 \dagger}$, Lin $\mathrm{He}^{2,3,4^{*}}$ and Yongyong Shi ${ }^{2,3,4^{*}}$

\begin{abstract}
The diagnosis of schizophrenia is currently based on the symptoms and bodily signs rather than on the pathological and physiological markers of the patient. In the search for new molecular targeted therapy medicines, and recurrence of early-warning indicators have become the major focus of contemporary research, because they improve diagnostic accuracy. Biomarkers reflect the physiological, physical and biochemical status of the body, and so have extensive applicability and practical significance. The ascertainment of schizophrenia biomarkers will help diagnose, stratify of disease, and treat of schizophrenia patients. The detection of biomarkers from blood has become a promising area of schizophrenia research. Recently, a series of studies revealed that, MiRNAs play an important role in the genesis of schizophrenia, and their abnormal expressions have the potential to be used as biomarkers of schizophrenia. This article presents and summarizes the value of peripheral blood miRNAs with abnormal expression as the biomarker of schizophrenia.
\end{abstract}

Keywords: Schizophrenia, Biomarkers, Peripheral MiRNAs, Significance and values

\section{Background}

Schizophrenia is a kind of brain dysfunction that can induce cognitive, emotional, mental and behavioral disorders. It affects about $1 \sim 1.5 \%$ of the world population [1]. Research findings showed that genetic factors were the main causes of schizophrenia and the heritability of schizophrenia can be as high as $80 \%$ [2]. It often occurs in young adults with symptoms so subtle that is difficult to identify and diagnose. The psychiatric diagnosis of schizophrenia specified in the latest [5th] edition of The Diagnostic and Statistical Manual of Mental Disorders (DSM) is based on symptoms rather than on pathophysiological and biochemical indicators [3]. The main symptoms of schizophrenia can be seen in Table 1. At present, the diagnoses of all mental disorders are based on signs and symptoms, rather than on pathophysiological and biochemical indicators [3]. Psychiatry has

\footnotetext{
*Correspondence: hekuanjun666@126.com; helinhelin@gmail.com; shiyongyong@gmail.com

†Equal contributors

'College of Life Science, Inner Mongolia University for Nationalities, Tongliao, Inner Mongolia 028043, People's Republic of China

${ }^{2}$ Bio-X Institutes, Key Laboratory for the Genetics of Developmental and

Neuropsychiatric Disorders (Ministry of Education), the Collaborative Innovation Center for Brain Science, Shanghai Jiao Tong University, Shanghai 200030,

People's Republic of China

Full list of author information is available at the end of the article
}

thus become one branch of medicine which does not rely on laboratory tests [4].

Biomarkers are substances that can reflect the physiological and biochemical status of an organism. They serve as indicators for pharmacological response in a normal biological process, case procedure or therapeutic intervention. The blood, urine, cerebrospinal fluid, autopsy specimens and tissue samples of schizophrenia patients provide rich gene and protein expressions under physiological and pathological conditions. A comprehensive analysis of these expressions is helpful in the identification of the biomarkers and in the determination of the therapeutic intervention measures and drug targets in the early diagnosis of the disease [5]. The detection of schizophrenia biomarkers aids prediction of the occurrence of the disease, accurate diagnosis, prognosis and treatment [6].

\section{MiRNAs and schizophrenia}

The etiology of schizophrenia is so complex that its causes are not yet fully understood. Both genetic and environmental factors are involved in the occurrence of schizophrenia [7, 8]. MicroRNAs (miRNAs) are a class of small, endogenous and noncoding single-stranded RNAs, 22-25 nucleotides in length, which participate in 
Table 1 The main symptoms of schizophrenia

\begin{tabular}{ll}
\hline Main symptons & Explaination \\
\hline Delusions & $\begin{array}{l}\text { These are false beliefs that are not } \\
\text { based in reality }\end{array}$ \\
Hallucinations & $\begin{array}{l}\text { Seeing or hearing things that } \\
\text { aren't there. }\end{array}$ \\
Disorganized thinking & $\begin{array}{l}\text { Using words and sentences that } \\
\text { don't make sense to others. }\end{array}$ \\
Extremely disorganized or & $\begin{array}{l}\text { Acting in an odd or repetitive way, } \\
\text { abnormal motor behavior }\end{array}$ \\
& $\begin{array}{l}\text { like walking in circles or writing all } \\
\text { and quiet for hours on end. }\end{array}$ \\
Negative symptoms & This refers to reduced or lack of \\
& ability to function normally. \\
\hline
\end{tabular}

the post-transcriptional regulation of gene expression. MiRNAs are estimated to regulate the translation of up to $60 \%$ of protein-coding genes [9]. Due to the differential target binding patterns, as many as 200 target genes may be regulated by a single miRNA [10]. It has been found that miRNAs were commonly present in a variety of organisms and are involved in almost all life processes, including development, differentiation, growth regulation and apoptosis [11].

It was confirmed that about $70 \%$ of human miRNAs express in the nervous system [12] and so are variously involved in the regulation of neural structure and function. For example, they play a role in the formation of dendrites and dendritic spines in axon growth as well as in neural developments and the maturation process [13]. Studies have demonstrated that they are also involved in the occurrence of neuropsychiatric disorders, and that their abnormal expressions could be treated as potential biomarkers [14, 15]. Miller et al. [14] (2010) and Sun [15] (2015) held the view that changes of miRNA expressions played a role in the genetic and biological mechanisms of neuropsychiatric diseases.

Epidemiological evidence suggests the etiology of schizophrenia may involve both genetic and environment factors. Thus, the environmental contribution to schizophrenia progression is through epigenetic mechanisms [16]. The study of mental illness from the perspective of epigenetics therefore informs and illuminates its pathogenesis. In the biological pathways of miRNA, the variation of miRNA target genes may also play a role in the occurrence of primarily schizophrenia. Beveridge et al. [17] (2012) considered that miRNAs influenced the occurrence of schizophrenia via the development of the central nervous system, gene expression regulation and other mechanisms. Since miRNAs are extensively regulated by transcription and are sensitive to changes in the biological pathway, miRNA abnormalities or mutations in cell may lead to neurological disorders including the pathophysiological changes of schizophrenia. Guo et al. [18]
(2010) confirmed that miR-195 was involved in a complex regulatory network by which the signaling pathways of the occurrence of schizophrenia were affected. The gene encoding miR-346 was located at the intron glutamate receptors ionic $\delta 1$ gene (GRID1), and the GRID1 gene is the susceptibility gene for schizophrenia [19]. MiR-30a-5p and miR-195 may regulate the gene expression of brain-derived neurotrophic factors $(B D N F)$, so it is suggested that miR195 may be involved in the pathogenesis of schizophrenia by regulating BDNF [20].

GWAS studies verify that rs1625579 located within miR-137 genes (1p21.3) was significantly associated with schizophrenia $\left(P=1.6 \times 10^{-11}\right)[21]$. The regulation target genes of miR-137 are CUB and Sushi multiple domains 1 gene (CSMD1), WW domain binding protein 1 like gene (C10orf26), calcium voltage-gated channel subunit alpha1 $\mathrm{C}$ gene $(C A C N A 1 C)$, transcription factor 4 gene (TCF4) and zinc finger protein 804A (ZNF804A), and these genes were confirmed to be genetic risk genes for schizophrenia [22-25].

Through bioinformatics and functional test methods, Valles et al. [26] (2014) confirmed again that five target genes that miR-137 regulated, including CACNA1C, TCF4 and $Z N F 804 A$, belong to the glucocorticoid receptordependent signal transduction network. CACNA1C gene in particularly has been repeatedly shown to be genetic susceptibility genes for schizophrenia in different types of population $[21,22,27,28]$.

\section{MiRNAs from peripheral blood as potential biomarkers for schizophrenia}

Many miRNAs are specifically expressed in cells or tissues, and their expression levels are also related to the pathological or physiological processes of the corresponding cells or tissues. Abnormal expression of miRNAs reflects the pathological state of the organism. Studies have found that the expression profiles of some miRNAs were significant different between the patient and the normal. Differentially expressed miRNAs are likely to be a noninvasive and accurate new biomarker for the diagnosis of disease. Although the etiology of schizophrenia is not certain, abnormal expressions of miRNAs are detected in brain tissues and peripheral blood on the part of schizophrenia patients. As biomarkers of tumors, miRNAs have been shown to be beneficial in clinical stratification, and to have even greater predictive value compared with mRNA [17]. The abnormally expressed miRNAs have been recently isolated from brain tissue [29-31], whole blood [32, 33], serum [34], plasma [35, 36] and PBMC [37], and recognized as potential biomarkers in the diagnosis of schizophrenia. The difficulties of conducting brain biopsies have consequences for obtaining abnormally expressed miRNAs for the purpose of prediction and diagnosis of a 
neurodegenerative disorder [38]. Since the expression profiles of miRNAs that are present in peripheral blood change with the changes of the body's physiological and pathological conditions [39], they have important clinical applications. Research investigating biomarkers of schizophrenia in peripheral blood is increasing. Particularly, more and more molecular detection techniques with high specificity and sensitivity are applied in the search for serum markers or genetic markers in peripheral blood.

Abnormally expressed miRNA has also been detected in the peripheral blood mononuclear cells and the plasma of the patients with schizophrenia. Gardiner et al. [37] (2011) analyzed miRNAs in peripheral blood mononuclear cells from 112 psychiatric patients and 76 non-psychotic patients under symptom control. They found that the 83 miRNAs expression in the 112 psychiatric patients was significantly lower than that of the non-psychotic group. 17 down-regulated miRNAs were from the imprinting area, DLK-DIO3 region of 14q32, which is closely associated with schizophrenia. Using
qRT-PCR, 7 miRNAs (miR-31, miR-431, miR-433, miR107, miR-134, miR-99b and miR-487b) were confirmed again to down-regulate.

Using microarray, Lai et al. [40] (2011) compared 30 schizophrenia patients with 30 controls and discovered differences in the miRNA expression of peripheral blood mononuclear leukocytes. Logistic regression analysis of the expression differences in the 7 miRNAs (hsamiR-34a, miR-449a, miR-564, miR-432, miR-548d, miR-572 and miR-652), revealed emotional retardation, language poverty and other neurocognitive dysfunction-associated symptoms. Shi et al. [34] (2012) found that miR-181b, miR-219-2-3p, miR-1308 and let-7 $\mathrm{g}$ in serum were up-regulated, while miRNA-195 was down-regulated. De la Morena et al. [32] (2013) investigated the miRNA expressions in the peripheral blood of patients with 22q11.2DS (22q11 micro-deletion syndrome), and found that 18 miRNAs were differently expressed, and that the miR-185 in the neurons of $90 \%$ to $95 \%$ of the 22 q $11.2 \mathrm{DS}$ patients was down-regulated.

Table 2 The list of miRNAs of peripheral blood that can be used as biomarkers of schizophrenia

\begin{tabular}{|c|c|c|c|c|}
\hline MiRNA & Biological functionality & $\begin{array}{l}\text { Direction associated } \\
\text { with schizophrenia }\end{array}$ & As Biomarkers & Author (Refs.) \\
\hline miRNA30e & Mitosis, cell division, etc & up-regulation & hsa-miRNA30e & Sun et al. [35] (2014) \\
\hline miR-130b & $\begin{array}{l}\text { nervous system development, neuron } \\
\text { differentiation, etc }\end{array}$ & up-regulation & hsa-miR-130b & Wei et al. [41](2015) \\
\hline miR-193a-3p & $\begin{array}{l}\text { neuron migration, nervous system } \\
\text { development, etc }\end{array}$ & up-regulation & hsa-miR-193a-3p & \\
\hline miR-30e & as above & up-regulation & \multirow{5}{*}{$\begin{array}{l}\text { hsa-miR-30e, hsa-miR-181b, } \\
\text { hsa-miR-34a, hsa-miR-346 } \\
\text { and hsa-miR-7 as a whole }\end{array}$} & \multirow[t]{5}{*}{ Sun et al. [36] (2015) } \\
\hline miR-181b & $\begin{array}{l}\text { central nervous system development, } \\
\text { aging, etc }\end{array}$ & up-regulation & & \\
\hline miR-34a & $\begin{array}{l}\text { neuron migration, generation of } \\
\text { neurons, organ regeneration, etc }\end{array}$ & up-regulation & & \\
\hline miR-346 & $\begin{array}{l}\text { response to stress, nervous system } \\
\text { development, learning or memory, etc }\end{array}$ & up-regulation & & \\
\hline miR-7 & $\begin{array}{l}\text { dendrite development, cellular } \\
\text { response to stress, etc }\end{array}$ & up-regulation & & \\
\hline miR-34a & as above & up-regulation & hsa-miR-34a & \multirow[t]{7}{*}{ Lai et al. [33] (2016) } \\
\hline miR-449a & $\begin{array}{l}\text { neuron apoptotic process, cellular } \\
\text { response to hypoxia, etc }\end{array}$ & up-regulation & hsa-miR-449a & \\
\hline miR-564 & $\begin{array}{l}\text { PI3K signaling networks, MAPK } \\
\text { signaling pathway, etc }\end{array}$ & up-regulation & hsa-miR-564 & \\
\hline $\operatorname{miR}-432$ & $\begin{array}{l}\mathrm{PI} 3 \mathrm{~K} / \mathrm{AKT} / \mathrm{mTOR} \text { signaling pathway, } \\
\text { cell proliferation and differentiation, etc }\end{array}$ & up-regulation & hsa-miR-432 & \\
\hline miR-548d & $\begin{array}{l}\text { nervous system development, positive } \\
\text { regulation of rho gtpase activity, etc }\end{array}$ & up-regulation & hsa-miR-548d & \\
\hline miR-572 & $\begin{array}{l}\text { cellular response to extracellular stimulus, } \\
\text { organ regeneration, response to } \\
\text { hyperoxia, etc }\end{array}$ & up-regulation & hsa-miR-572 & \\
\hline miR-652 & $\begin{array}{l}\text { regulation of energy homeostasis, } \\
\text { dendrite morphogenesis, etc }\end{array}$ & up-regulation & hsa-miR-652 & \\
\hline miR-30a-5p & $\begin{array}{l}\text { response to hypoxia, neuron migration, } \\
\text { immune response, etc }\end{array}$ & down regulation & EGR1-miR-30a-5p-NEUROD1 axis & Liu et al. [42] (2017) \\
\hline
\end{tabular}


By using real-time quantitative PCR, Sun et al. [35] (2014) analyzed the expression profiles of nine schizophreniarelated miRNAs in peripheral blood plasma and mononuclear cells, and confirmed that miRNA30e specificity in plasma could be used as a biomarker for diagnosis of schizophrenia. Wei et al. [41] (2015) demonstrated by using high throughput sequencing and RT- qPCR assays that miR-130b and miR-193a$3 p$ serve as biomarkers for the diagnosis of schizophrenia. Using qPCR, Sun et al. [36] (2015) analyzed the expression profiles of 10 miRNAs in plasma from 61 patients with schizophrenia, 62 normal controls, and 25 patients taking antipsychotic drugs for 6 weeks. It was found that miR-30e, MiR-181b, miR-34a, miR346 and miR-7 as a whole can be used as a noninvasive diagnostic marker for schizophrenia, and that miR-132, miR-181b, miR-30e and miR-432 can all be used as indicators of schizophrenia symptom improvement, drug efficacy and prognosis.

Using qPCR, Lai et al. [33] (2016) analyzed the expressions of 7 miRNAs (hsa-miR-34a, miR-449a, miR-564, miR-432, miR-548d, miR-572, miR-542 and MiR-652) that had previously been identified as potential biomarkers for schizophrenia in acute disease. After 2 months of hospitalization and symptomatic relief, the expressions of the 7 miRNAs in peripheral blood of patients did not change and that their expressions in peripheral blood were stable as schizophrenia diagnostic biomarkers. Using RT-qPCR technology, Liu et al. [42] (2017), suggested that the EGR1-miR-30a-5pNEUROD1 axis might serve as a promising biomarker for diagnosis and treatment monitoring for patients in an acute psychotic state.

The above tests confirm that miRNA profiling in peripheral blood can be used to determine biomarkers for schizophrenia and related subtypes of mental illness. MiRNAs of peripheral blood that can be biomarkers of schizophrenia can be seen in Table 2. It may help to elucidate the etiology of schizophrenia with ambiguous clinical symptoms and identifies potential differences between psychiatric disorders with similar, easily confusable symptoms [43].

\section{Conclusions}

Progress in the foregoing highlights the potential significance of miRNA as a diagnostic marker of schizophrenia. MiRNA expression profiles from peripheral blood not only are potential schizophrenia biomarkers but also have association with schizophrenia subtypes. The study of miRNA expression profiles in peripheral blood is of far reaching significance because it provides the basis for the early detection of schizophrenia, disease stratification and the prediction of drug response and side effects.
It enables further study of the regulatory mechanisms of gene expression, their relationship with the disease, their proteins expression profiles, and the molecular mechanisms involved in the pathogenesis of schizophrenia, and it will lead to the elucidation of the complex etiology of schizophrenia. Studies of the molecular mechanisms of the occurrence and development of schizophrenia, its specificity, especially of the early warning and diagnostic biomarkers obtained directly from the blood, have been an important and urgent priority in schizophrenia research. Ongoing improvements in the detection, assessment and intervention strategies for miRNA are promoting the development of the diagnosis and treatment of schizophrenia, and facilitating miRNA applications in other disease diagnoses and treatments.

\section{Abbreviations}

22q11DS: 22q11 micro-deletion syndrome; BDNF: Brain-derived neurotrophic factors; C10orf26: WW domain binding protein 1 like gene; CACNA1C: Calcium voltage-gated channel subunit alpha1 C gene; CSMD1: CUB and sushi multiple domains 1 gene; DSM: The diagnostic and statistical manual of mental disorders; GRID1: Intron glutamate receptors ionic $\delta 1$ gene; MiRNAs: MicroRNAs;

TCF4: Transcription factor 4; ZNF804A: Gene and zinc finger protein 804A

\section{Acknowledgements}

We are deeply grateful to Eric Smithson of Ningbo University for the revision and correction of the paper.

\section{Funding}

This work was supported by the Natural Science Foundations of China (No 81501151, No 81660234), the Natural Science Foundation of Inner Mongolia (No 2015BS0808), the Doctor Scientific Research Foundation of Inner Mongolia University for Nationalities (No BS331), the National Key Basic Research Program of China (973 Program) (2015CB559100), the Program of Shanghai Academic Research Leader (15XD1502200), National Program for Support of Top-Notch Young Professionals, Shanghai Key Laboratory of Psychotic Disorders (13dz2260500), and Inner Mongolia Science and Technology Plan (20130612).

Availability of data and materials

Data sharing not applicable to this article as no datasets were generated or analysed during the current study.

Authors' contributions

$\mathrm{KH}, \mathrm{CG}, \mathrm{YS}$ and $\mathrm{HL}$ conceived the study and wrote the manuscript. All authors read and approved the final manuscript.

Ethics approval and consent to participate

Not applicable.

Consent for publication

Not applicable.

Competing interests

The authors declare that they have no competing interests.

\section{Publisher's Note}

Springer Nature remains neutral with regard to jurisdictional claims in published maps and institutional affiliations.

\section{Author details}

${ }^{1}$ College of Life Science, Inner Mongolia University for Nationalities, Tongliao, Inner Mongolia 028043, People's Republic of China. Bio-X Institutes, Key Laboratory for the Genetics of Developmental and Neuropsychiatric Disorders (Ministry of Education), the Collaborative Innovation Center for Brain Science, Shanghai Jiao Tong University, Shanghai 200030, People's Republic of China. ${ }^{3}$ Shanghai Key Laboratory of Psychotic Disorders, Shanghai Mental Health 
Center, Shanghai Jiao Tong University School of Medicine, Shanghai 200030, People's Republic of China. ${ }^{4}$ Institute of Neuropsychiatric Science and Systems Biological Medicine, Shanghai Jiao Tong University, Shanghai 200042, People's Republic of China.

\section{Received: 3 April 2017 Accepted: 16 August 2017}

Published online: 29 August 2017

\section{References}

1. Mueser KT, Mcgurk SR. Schizophrenia. Lancet. 2004;363(9426):2063-72.

2. van Os J, Kapur S. Schizophrenia. Lancet. 2009;374(9690):635-45.

3. Vargas G. Biomarkers in schizophrenia. Biomark Med. 2014;8(1):1-3.

4. Kapur S, Phillips AG, Insel TR. Why has it taken so long for biological psychiatry to develop clinical tests and what to do about it? Mol Psychiatry. 2012;17(12): 1174-9.

5. Li S, Zhang A, Lin H. Progress of GWAS and Translational Medicine for Schizophrenia in China. china science:life science. 2013;01:31-8.

6. Pickard BS. Schizophrenia biomarkers: translating the descriptive into the diagnostic. J Psychopharmacol. 2015;29(2):138-43.

7. Messias EL, Chen C, Eaton WW. Epidemiology of schizophrenia: review of findings and myths. Psychiatr Clin N Am. 2007;30(3):323-38.

8. Berry N, Jobanputra $\mathrm{V}, \mathrm{Pal} \mathrm{H}$. Molecular genetics of schizophrenia: a critical review. J Psychiatry Neurosci. 2003;28(6):415-29.

9. Lewis BP, Burge CB, Bartel DP. Conserved seed pairing, often flanked by adenosines, indicates that thousands of human genes are microRNA targets. Cell. 2005;120(1):15-20.

10. Krek A, Grun D, Poy MN, Wolf R, Rosenberg L, Epstein EJ, et al. Combinatorial microRNA target predictions. Nat Genet. 2005;37(5):495-500.

11. Bartel DP. MicroRNAs: genomics, biogenesis, mechanism, and function. Cell. 2004;116(2):281.

12. Nowak JS, Michlewski G. miRNAs in development and pathogenesis of the nervous system. Biochem Soc Trans. 2013;41(4):815-20.

13. Wang J, Wang Y, Yang J, Huang Y. microRNAs as novel biomarkers of schizophrenia (review). Experimental and Therapeutic Medicine. 2014;8(6): 1671-6.

14. Miller $\mathrm{BH}$, Wahlestedt C. MicroRNA dysregulation in psychiatric disease. Brain Res. 2010;1338:89-99.

15. Sun E, Shi Y. MicroRNAs: small molecules with big roles in neurodevelopment and diseases. Exp Neurol. 2015:268:46-53.

16. Popov NT, Stoyanova VK, Madzhirova NP, Vachev TI. Epigenetic aspects in schizophrenia etiology and pathogenesis. Folia Med (Plovdiv). 2012;54(2):12-6.

17. Beveridge NJ, Cairns MJ. MicroRNA dysregulation in schizophrenia. Neurobiol Dis. 2012;46(2):263-71.

18. Guo A, Sun J, Jia P, Zhao Z. A novel microRNA and transcription factor mediated regulatory network in schizophrenia. BMC Syst Biol. 2010;4(1):10.

19. Zhu Y, Kalbfleisch T, Brennan MD, Li Y. A MicroRNA gene is hosted in an intron of a schizophrenia-susceptibility gene. Schizophr Res. 2009;109(1-3):86-9.

20. Mellios N, Huang HS, Baker SP, Galdzicka M, Ginns E, Akbarian S. Molecular determinants of dysregulated GABAergic gene expression in the prefrontal cortex of subjects with schizophrenia. Biol Psychiatry. 2009;65(12):1006-14.

21. Ripke S, Sanders AR, Kendler KS, Levinson DF, Sklar P, Holmans PA, et al. Genome-wide association study identifies five new schizophrenia loci. Nat Genet. 2011;43(10):969-76.

22. Hamshere ML, Walters JTR, Smith R, Richards AL, Green E, Grozeva D, et al. Genome-wide significant associations in schizophrenia to ITIH3/4, CACNA1C and SDCCAG8, and extensive replication of associations reported by the schizophrenia PGC. Mol Psychiatry. 2012;18(6):708-12.

23. Navarrete K, Pedroso I, De Jong S, Stefansson H, Steinberg S, Stefansson K, et al. TCF4 (e2-2; ITF2): a schizophrenia-associated gene with pleiotropic effects on human disease. Am J Med Genet B Neuropsychiatr Genet. 2013;162B(1):1-16

24. Kwon E, Wang W, Tsai LH. Validation of schizophrenia-associated genes CSMD1, C10orf26, CACNA1C and TCF4 as miR-137 targets. Mol Psychiatry. 2013;18(1):11-2.

25. Kim AH, Parker EK, Williamson V, Mcmichael GO, Fanous AH, Vladimirov VI. Experimental validation of candidate schizophrenia gene ZNF804A as target for hsa-miR-137. Schizophr Res. 2012;141(1):60-4

26. Valles A, Martens GJ, De Weerd P, Poelmans G, Aschrafi A. MicroRNA-137 regulates a glucocorticoid receptor-dependent signalling network: implications for the etiology of schizophrenia. J Psychiatry Neurosci. 2014;39(5):312-20.
27. He K, An Z, Wang Q, Li T, Li Z, Chen J, et al. CACNA1C, schizophrenia and major depressive disorder in the Han Chinese population. Br J Psychiatry. 2014;204(1):36-9.

28. Guan F, Zhang B, Yan T, Li L, Liu F, Li T, et al. MIR137 gene and target gene CACNA1C of miR-137 contribute to schizophrenia susceptibility in Han Chinese. Schizophr Res. 2014;152(1):97-104.

29. Santarelli DM, Beveridge NJ, Tooney PA, Cairns MJ. Upregulation of dicer and MicroRNA expression in the dorsolateral prefrontal cortex Brodmann area 46 in schizophrenia. Biol Psychiatry. 2011;69(2):180-7.

30. Moreau MP, Bruse SE, David-Rus R, Buyske S, Brzustowicz LM. Altered MicroRNA expression profiles in postmortem brain samples from individuals with schizophrenia and bipolar disorder. Biol Psychiatry. 2011;69(2):188-93.

31. Mor E, Kano S, Colantuoni C, Sawa A, Navon R, Shomron N. MicroRNA-382 expression is elevated in the olfactory neuroepithelium of schizophrenia patients. Neurobiol Dis. 2013;55:1-10.

32. de la Morena MT, Eitson JL, Dozmorov IM, Belkaya S, Hoover AR, Anguiano $\mathrm{E}$, et al. Signature MicroRNA expression patterns identified in humans with 22q11.2 deletion/DiGeorge syndrome. Clin Immunol. 2013;147(1):11-22.

33. Lai CY, Lee SY, Scarr E, Yu YH, Lin YT, Liu CM, et al. Aberrant expression of microRNAs as biomarker for schizophrenia: from acute state to partial remission, and from peripheral blood to cortical tissue. Transl Psychiatry. 2016:6:e717.

34. Shi W, Du J, Qi Y, Liang G, Wang T, Li S, et al. Aberrant expression of serum miRNAs in schizophrenia. J Psychiatr Res. 2012;46(2):198-204.

35. Sun $X Y$, Lu J, Zhang L, Song $H T$, Zhao L, Fan $H$ M, etal. Aberrant microRNA expression in peripheral plasma and mononuclear cells as specific bloodbased biomarkers in schizophrenia patients. J Clin Neurosci. 2015;22(3):5704.

36. Sun XY, Zhang J, Niu W, Guo W, Song HT, Li HY, et al. A preliminary analysis of microRNA as potential clinical biomarker for schizophrenia. Am J Med Genet B Neuropsychiatr Genet. 2015;168B(3):170-8.

37. Gardiner E, Beveridge NJ, Wu JQ, Carr V, Scott RJ, Tooney PA, et al. Imprinted DLK1-DIO3 region of 14q32 defines a schizophrenia-associated miRNA signature in peripheral blood mononuclear cells. Mol Psychiatry. 2011; 17(8):827-40.

38. Nuzzo D, Picone P, Caruana L, Vasto S, Barera A, Caruso C, et al. Inflammatory mediators as biomarkers in brain disorders. Inflammation. 2014;37(3):639-48.

39. Alevizos I, IIlei GG. MicroRNAs as biomarkers in rheumatic diseases. Nat Rev Rheumatol. 2010;6(7):391-8.

40. Lai C, Yu S, Hsieh MH, Chen C, Chen H, Wen C, et al. MicroRNA expression aberration as potential peripheral blood biomarkers for schizophrenia. PLoS One. 2011;6(6):e21635

41. Wei H, Yuan Y, Liu S, Wang C, Yang F, Lu Z, et al. Detection of circulating miRNA levels in schizophrenia. Am J Psychiatry. 2015;172(11):1141-7.

42. Liu S, Zhang F, Shugart YY, Yang L, Li X, Liu Z, et al. The early growth response protein 1-miR-30a-5p-neurogenic differentiation factor 1 axis as a novel biomarker for schizophrenia diagnosis and treatment monitoring. Trans| Psychiatry. 2017;7(1):e998.

43. Kalia M, Costa E, Silva J. Biomarkers of psychiatric diseases: current status and future prospects. Metabolism. 2015;64(3):S11-5.

\section{Submit your next manuscript to BioMed Central and we will help you at every step:}

- We accept pre-submission inquiries

- Our selector tool helps you to find the most relevant journal

- We provide round the clock customer support

- Convenient online submission

- Thorough peer review

- Inclusion in PubMed and all major indexing services

- Maximum visibility for your research

Submit your manuscript at www.biomedcentral.com/submit 